
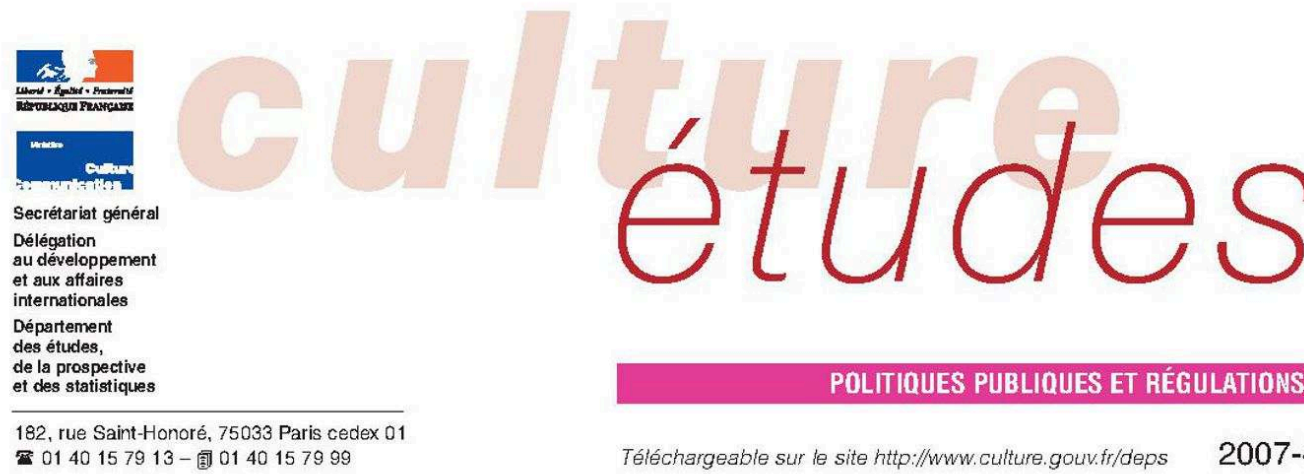

\title{
Économies des droits d'auteur I. Le livre
}

\section{Christian Robin*}

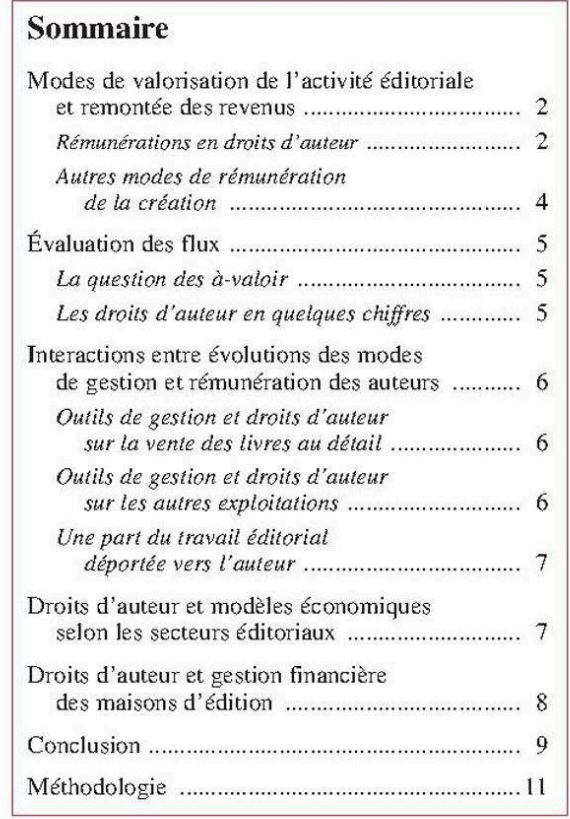

\author{
Avant-propos \\ L'économie de l'auteur de littérature au sein de la \\ filière de l'édition, la plus importante des industries \\ culturelles, est un modele historique canonique.
}

Pourtant, l'économie du droit d'auteur dans le livre s'est renouvelée et transformée : autour de la rémunération proportionnelle aux ventes des auteurs, s'ajoutent d'autres modes de rémunération; des catégories connexes des auteurs sont titulaires de droits d'auteur; des modes de gestion spécifiques et des exploitations plus nombreuses se sont développés.

Cette économie du droit d'auteur demeure un modele. Mais sous l'effet des exigences industrielles et financières, de l'évolution des technologies de l'édition, de la nature de l'auteur (ou des auteurs) comme des publications, elle poursuit une mutation apparemment lente mais déjà profonde.

P. C.

\footnotetext{
* Mâtre de conférences en sciences de l'information et de la communication à l'université Paris 13 et directeur de Fomedi, cabinet de conseil et de formation. Publications récentes: "Precio y cultura. El precio unico del libro en Europa», Texturas n० 1, décembre 2006; Christian RoBin et Bertrand Legendre (sous la dir. de), Figures de l'éditeur, Paris, Nouveau monde Éditions, 2005 (actes de colloque); « Numerisation du livre, quelle influence sur les contenus? ?, Communication et langages, $\mathrm{n}^{\circ} 145$, septembre 2005 ; «Les éditeurs publient-ils trop de livres? », Communication et langages, $\mathrm{n}^{\circ} 143$, mars 2005, etc
} 


\section{Économies des droits d'auteur - I}

Le livre

\section{Christian Robin}

Éditeur : Département des études, de la prospective et des statistiques

Lieu d'édition : Paris

Année d'édition : 2007

Date de mise en ligne : 8 juillet 2015

Collection : Culture études

ISBN électronique : 9782111398757

\section{opentitition ebooks}

http://books.openedition.org

\section{Édition imprimée}

Date de publication : 1 décembre 2007

Nombre de pages : 12

\section{Référence électronique}

ROBIN, Christian. Économies des droits d'auteur - I: Le livre. Nouvelle édition [en ligne]. Paris :

Département des études, de la prospective et des statistiques, 2007 (généré le 25 avril 2021).

Disponible sur Internet : <http://books.openedition.org/deps/414>. ISBN : 9782111398757.

(C) Département des études, de la prospective et des statistiques, 2007

Creative Commons - Attribution - Pas d'Utilisation Commerciale 3.0 non transposé - CC BY-NC 3.0 


\section{Économies des droits d'auteur I. Le livre}

\section{Christian Robin*}

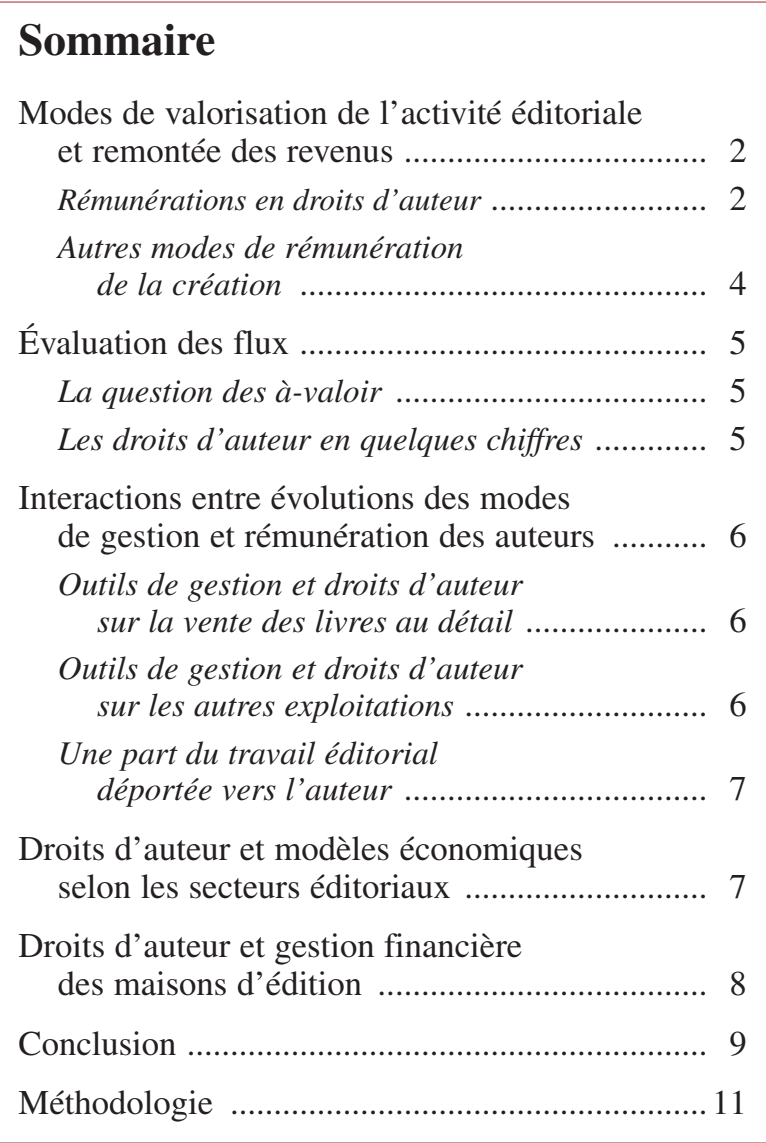

\begin{abstract}
Avant-propos
L'économie de l'auteur de littérature au sein de la filière de l'édition, la plus importante des industries culturelles, est un modèle historique canonique.
\end{abstract}

Pourtant, l'économie du droit d'auteur dans le livre s'est renouvelée et transformée : autour de la rémunération proportionnelle aux ventes des auteurs, s'ajoutent d'autres modes de rémunération; des catégories connexes des auteurs sont titulaires de droits d'auteur; des modes de gestion spécifiques et des exploitations plus nombreuses se sont développés.

Cette économie du droit d'auteur demeure un modèle. Mais sous l'effet des exigences industrielles et financières, de l'évolution des technologies de l'édition, de la nature de l'auteur (ou des auteurs) comme des publications, elle poursuit une mutation apparemment lente mais déjà profonde.

\footnotetext{
*Maître de conférences en sciences de l'information et de la communication à l'université Paris 13 et directeur de Formedi, cabinet de conseil et de formation. Publications récentes: «Precio y cultura. El precio unico del libro en Europa », Texturas n 1, décembre 2006; Christian Robin et Bertrand Legendre (sous la dir. de), Figures de l'éditeur, Paris, Nouveau monde Éditions, 2005 (actes de colloque) ; « Numérisation du livre, quelle influence sur les contenus ?», Communication et langages, $\mathrm{n}^{\circ} 145$, septembre 2005 ; « Les éditeurs publient-ils trop de livres? », Communication et langages, $\mathrm{n}^{\circ}$ 143, mars 2005, etc.
} 
La notion de propriété littéraire et artistique est intrinsèque à la filière du livre : une grande partie de la législation d'origine sur ces questions s'est développée à partir de problématiques liées au livre imprimé, et de nombreux articles du Code de la propriété intellectuelle (CPI) traitent du contrat d'édition.

Avec un très grand nombre de titres nouveaux ou nouvelles éditions publiés chaque année - près de $60000^{1}$ - et une très forte progression de ce nombre alors que les ventes de livres croissent faiblement, se pose la question des modes de rentabilisation de cette production.

Les évolutions techniques et les usages interrogent par ailleurs les modèles économiques des éditeurs. Dans certains cas, la vente de livres n'est plus qu'un élément d'une offre plus large de services ; d'autre part des modes d'accès - prêt en bibliothèque, photocopie ou $\mathrm{Web}-$, toujours plus variés ne font pas l'objet d'une transaction monétaire. La question des modes de rémunération des auteurs et des éditeurs dans ces nouveaux modèles, et en particulier dans le cadre de stratégies d'exploitation multisupport, se pose donc avec encore plus d'acuité.

Pour autant, les pratiques ne sont pas révolutionnées par l'irruption de nouvelles techniques ni par l'évolution du contexte et il s'agit donc pour l'essentiel d'usages très « installés ».

Par rapport à d'autres secteurs des industries culturelles, le rôle des sociétés d'auteurs est faible dans l'industrie du livre. Toutefois, des organismes spécifiques ont été mis en place pour gérer les droits issus des «nouvelles » exploitations.

\section{MODES DE VALORISATION \\ DE L'ACTIVITÉ ÉDITORIALE \\ ET REMONTÉE DES REVENUS}

Aux quatre grands modes traditionnels de valorisation dans la filière livre - vente du livre au détail, vente de livres fabriqués à façon, cession de droits d'exploitation, produit d'activités connexes comme la vente d'espaces publicitaires, prestations de services relatifs aux contenus dont l'éditeur est détenteur - s'est ajoutée, ces dernières décennies, la rémunération d'exploitations s'apparentant à une licence légale ${ }^{2}$.

Nombreux sont les acteurs et les modes de rémunération auxquels chacun a droit (voir figure 1). Cependant, les différents intervenants à la création sont rémunérés par l'éditeur non seulement en droits d'auteur mais aussi en salaires ou en prestations de services. Entre ces différents modes de rémunération, la frontière est floue. Les usages de la propriété littéraire sont en effet restrictifs ou extensifs suivant les acteurs et selon les modalités d'organisation, comme le montre l'exemple du maquettiste, rémunéré différemment selon qu'il est salarié ou pas de la maison d'édition et selon qu'il fait un travail de création ou d'exécution.

Ces observations permettent de montrer les limites des informations collectées sur les montants de droits d'auteur ou sur les contrats d'auteur, puisque une part non négligeable de la création originale ne passe pas par des auteurs sous contrat et de nombreux travaux connexes à la création sont effectués dans le cadre de contrats assimilés aux contrats d'auteur et ce pour des questions essentiellement organisationnelles.

\section{Rémunérations en droits d'auteur}

Les rémunérations sous forme de droits d'auteur sont, pour une part, calculées en pourcentage des ventes, en application de la règle générale définie par le CPI et, pour l'autre, en droits forfaitaires indépendants des ventes ${ }^{3}$. C'est ainsi que sont rémunérés les auteurs de livres - texte ou illustrations clairement identifiés mais aussi d'autres intervenants comme les directeurs d'ouvrage ou de collection $^{4}$ et les packagers ${ }^{5}$ qui réalisent tout ou partie du travail éditorial.

\section{Les droits proportionnels}

\section{Le cas général}

Le contrat, qui lie l'auteur à l'éditeur, définit le mode de calcul des droits. Entrent en compte les paramètres suivants :

\footnotetext{
1. Source Électre.

2. Possibilité d'utilisation d'une œuvre sans autorisation préalable du titulaire des droits.

3. Voir Philippe Schuwer, Traité pratique d'édition, Paris, Éditions du Cercle de la librairie, 2002.

4. Les directeurs d'ouvrage ou apporteurs de projet réalisent un travail éditorial plus ou moins poussé ; les directeurs de collection font comme les précédents mais au niveau d'une collection de livres.

5. Le packager peut réaliser une prestation très complète partant d'un projet dont lui ou l'éditeur a été à l'initiative jusqu'à la fourniture des fichiers des livres à l'imprimeur et même le suivi de la fabrication (impression et façonnage) du livre ou, au contraire, un suivi éditorial partiel jouant le rôle de secrétaire d'édition externe. Dans tous les cas, il aura une délégation de réalisation d'ouvrage.
} 


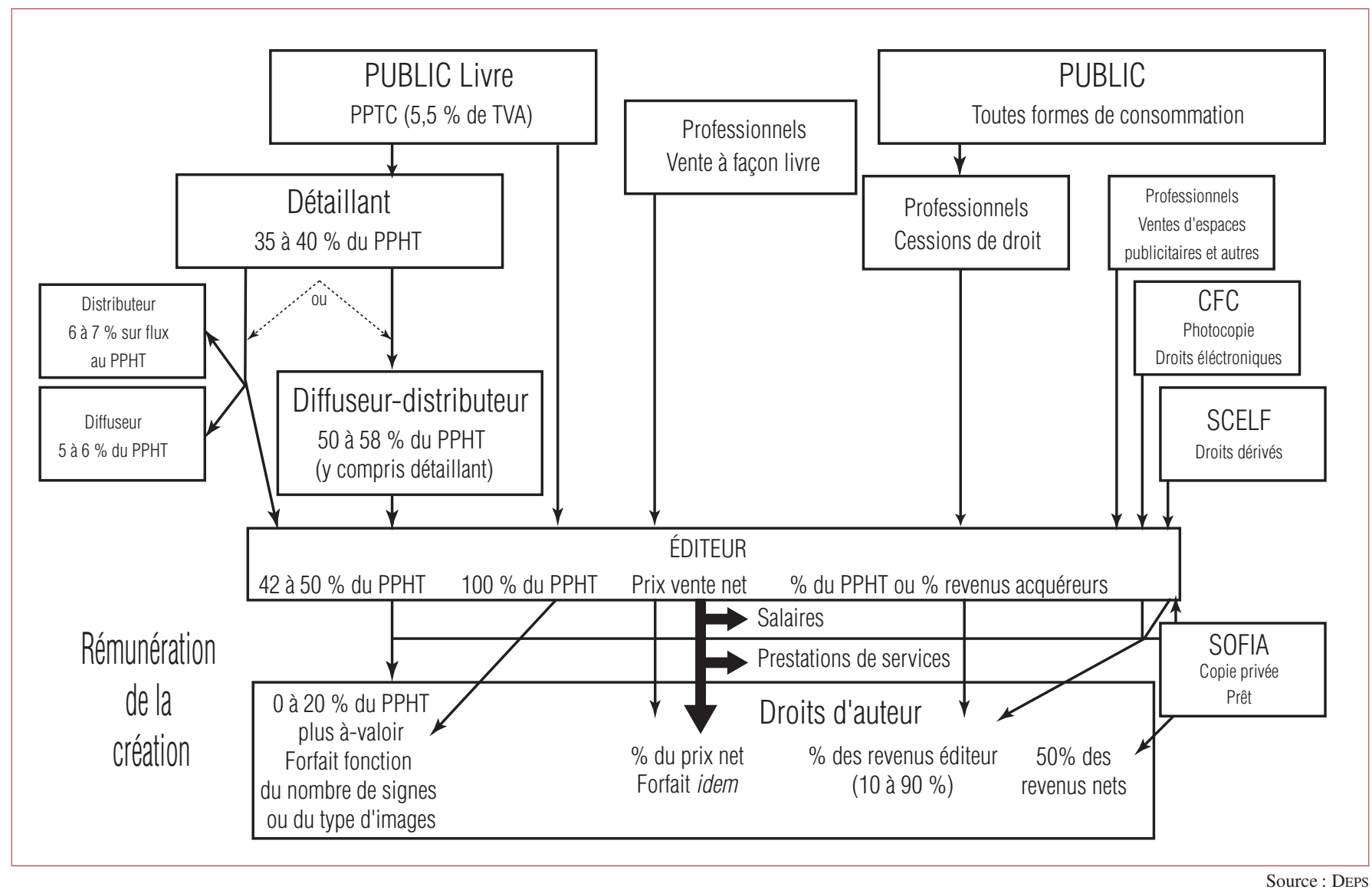

- le taux des droits, qui peut varier en fonction du nombre d'exemplaires vendus selon une échelle progressive (par exemple $6 \%$ jusqu'à 5000 exemplaires vendus, $8 \%$ de 5001 à 10000 et $10 \%$ au-delà);

- la base en valeur (généralement le prix public hors taxes, PРHT) et en quantité (le nombre d'exemplaires vendus), sur laquelle le pourcentage va être calculé ;

- les éventuels abattements sur ces bases, c'est-àdire une diminution de la valeur de référence, prix ou quantité. Les éditeurs appliquent, par exemple, un abattement sur le PPHT (15 à $30 \%$ ) en fonction de l'existence d'une reliure (le complément de prix lié à la reliure n'est pas supposé occasionner des droits). Ils pratiquent de même pour les ventes hors Europe car les coûts d'acheminement sont très élevés (souvent $50 \%$ ), ou pour les ventes réalisées avec une remise supérieure à un niveau préétabli. Les droits, enfin, ne sont pas calculés sur les envois de spécimens faits à la presse ou à d'autres personnes, ni sur les exemplaires détruits.

Souvent, une avance sur droits - ou à-valoir - est prévue dans le contrat: dans ce cas, l'auteur ne commence à percevoir ses droits sur les ventes qu'une fois l'à-valoir couvert. Cette avance est acquise une fois pour toutes sous certaines conditions relatives à l'acceptation du manuscrit et son montant varie en fonction de la notoriété de l'auteur, de quelques centaines à plusieurs millions d'euros. Dans le cas d'un fort à-valoir, il peut arriver que les droits calculés sur les ventes ne le couvrent jamais.

Les taux des auteurs «primaires » sont extrêmement variés. Pour un auteur unique, le taux réel, c'est-à-dire déduction faite des abattements, peut aller de 3 à $18 \%$ du PPHT. Dans le cas d'une collaboration, les divers auteurs se partagent ces pourcentages. Les traducteurs littéraires perçoivent un faible pourcentage du PPHT (de 0,5 à $2 \%$ ) mais ont un à-valoir qui dépend du volume de travail calculé comme pour les droits d'auteur forfaitaires. Le tarif de leur prestation varie en fonction de la langue d'origine du texte. Les directeurs d'ouvrage ou de collection reçoivent de 1 à $2 \%$ du PPHT et souvent un petit à-valoir (quelques centaines d'euros).

\section{Deux situations spécifiques}

Deux situations particulières, celle des directeurs d'ouvrage ou de collection et celle des packagers, méritent d'être développées. 
Les directeurs d'ouvrages ou de collections

Certains peuvent avoir un contrat s'apparentant en tout point à celui d'un auteur - cession de droits pour la durée de la propriété littéraire, droits secondaires sur toutes éditions - et pourraient donc, si l'on s'en tenait uniquement au contrat, revendiquer un droit moral, ce qui peut se justifier pour les ouvrages collectifs publiés sous la direction d'une personnalité, mais moins dans le cas où ils apportent simplement un projet.

D'autres sont rémunérés selon un contrat dit « viager» où la rémunération ne leur est due que jusqu'à leur mort: la notion de droits d'auteur au sens du CPI n'est donc pas présente et, pourtant, les systèmes comptables des éditeurs considèrent ces sommes comme des droits d'auteur.

Ces deux formes contractuelles peuvent être utilisées par un même éditeur.

\section{Les packagers}

Leur rémunération dépend de la nature de leur travail mais s'apparente d'un côté à celle des auteurs, de l'autre à celle des prestataires de services. Les modalités contractuelles dépendent du type d'intervention qu'ils réalisent ainsi que du souhait de l'éditeur de se protéger plus ou moins fortement. Que sa prestation soit très complète ou qu'elle ne soit qu'un suivi éditorial partiel (il joue alors le rôle de secrétaire d'édition externe), le packager a une délégation de réalisation d'ouvrage.

La mission inclut ou non la signature de contrats d'auteur à son nom. Si le packager conclut les contrats d'auteur, il cède ses droits à l'éditeur selon un contrat classique d'édition avec un pourcentage sur les ventes et un à-valoir, à charge pour lui de rémunérer les auteurs.

Ce cas, fréquent pour les œuvres collectives, peut aussi se produire pour des ouvrages qui comportent des auteurs «principaux » uniques ou en collaboration. Le packager se retrouve alors dans la situation d'un éditeur cédant ses droits et prélevant donc une partie des droits des auteurs premiers. Nombreux sont les éditeurs se trouvant dans cette situation qui souhaitent conclure directement les contrats avec les auteurs afin de limiter les risques que le packager ne se transforme en éditeur.

Le packager est rémunéré de deux manières pour son travail (hors les contrats d'auteur) : il perçoit des « droits d'auteur » et le remboursement des frais techniques. Le contrat «d'auteur» pour la participation à la conception de l'ouvrage peut être forfaitaire ou comporter des clauses d'intéressement aux ventes, avec des taux allant de $1 \%$ pour une simple direction d'ouvrage à $10 \%$ pour une intervention globale, et plus généralement 5 à $6 \%$.

Le risque encouru dans le cas d'un contrat d'auteur conclu par un packager, c'est qu'à la disparition de ce dernier, l'éditeur exploite un ouvrage dont les droits sont en déshérence.

\section{Les droits forfaitaires}

Pour le texte, les rémunérations sous la forme de droits forfaitaires concernent habituellement les contributions ponctuelles (préfaces ou postfaces comprises) et les ouvrages avec de multiples auteurs - « œuvres collectives » et «œuvres en collaboration ». Le calcul de la rémunération se fait, sauf dans le cas des préfaces et postfaces, sur la base d'un feuillet standard de 1500 ou 1800 signes, avec des tarifs variant de 20 à 50 euros le feuillet. Toutefois, lorsqu'il s'agit de textes réutilisés pour d'autres ouvrages et encore plus de rééditions du même titre, il est rare que les éditeurs versent des rémunérations complémentaires, en toute contradiction avec ce que prescrit la loi.

En ce qui concerne l'iconographie, elle est essentiellement payée en droits forfaitaires selon une tarification beaucoup plus complexe que pour le texte puisqu'elle dépend du sujet reproduit (personne, œuvre elle-même protégée ou non) et de l'auteur de cette image (photographie, illustration, carte, etc.). Pour les photographies, elle dépend également du tirage du livre.

Par ailleurs, certains travaux sont parfois payés en droits d'auteur forfaitaires alors que leur nature ne devrait pas s'y prêter, en particulier certaines prestations d'édition free-lance.

\section{Autres modes de rémunération de la création}

Les travaux de création originale par nature peuvent être rémunérés en salaires ou prestations de services : en particulier, la création de maquettes ou de cartes, mais aussi certains travaux d'écriture pour des ouvrages collectifs (lexicographie ou commentaires de texte juridique par exemple) ou de réécriture menés par un secrétaire d'édition. La rémunération des activités créatives du personnel éditorial fluctue souvent au gré des rapports de force internes et des jurisprudences. 


\begin{tabular}{|lrrrrrrrr|} 
& \multicolumn{1}{c}{ En millions d'euros } \\
\hline & 1990 & 1995 & $\mathbf{2 0 0 0}$ & $\mathbf{2 0 0 1}$ & $\mathbf{2 0 0 2}$ & $\mathbf{2 0 0 3}$ & $\mathbf{2 0 0 4}$ & $\mathbf{2 0 0 5}$ \\
Chiffre d'affaires (hors Clubs) & 2038 & 2154 & 2285 & 2353 & 2461 & 2540 & 2594 & 2746 \\
$\begin{array}{l}\text { dont cessions de droits } \\
\text { Droits d'auteur }\end{array}$ & 193 & 209 & 263 & 285 & 295 & 355 & 102 & 427 \\
\hline
\end{tabular}

\section{ÉVALUATION DES FLUX}

Pour analyser la place qu'occupent les droits d'auteur dans la filière livre et le rôle économique qu'ils y jouent, il faut d'abord évaluer ce secteur dont l'économie repose sur les droits d'auteur. Or, les données disponibles sur le marché du livre en France varient considérablement : il serait d'environ cinq milliards d'euros (au prix public) selon le syndicat national de l'édition (SNE), alors qu'une étude du DePs évaluait à 5,3 milliards d'euros le chiffre d'affaires ${ }^{6}$ de la filière hors activité librairie, chiffre beaucoup plus élevé que ce qu'indiquent les statistiques du SNE (voir tableau 1).

Cette différence ne pouvant pas être expliquée par le seul fait d'intégrer ou pas certaines activités, pas plus que par des différences d'échantillon, ce sont donc les statistiques du SNE qui sont ici utilisées. Elles ont l'intérêt de constituer une série longue et de comporter une évaluation des droits d'auteur.

\section{La question des à-valoir}

Les droits d'auteur déclarés au SNE comprennent en général les droits proportionnels, les droits forfaitaires - y compris les droits photographiques et la contribution diffuseur de $1 \%$ versée à l'AgESSA ${ }^{7}$. En revanche, se pose la question des àvaloir: pour de nombreuses maisons d'édition en effet, ils ne passent pas directement dans les comptes auteurs. Ils n'apparaissent dans ceux-ci que pour le montant couvert par les droits calculés sur les ventes, le reste faisant l'objet d'une dépréciation et venant donc en charge au fur et à mesure que celle-ci est constatée. L'à-valoir non couvert est ensuite passé en perte.
Ce système induit un long décalage entre le versement et le constat comptable des droits : un àvaloir peut en effet rester déprécié pendant toute la durée de la propriété littéraire si l'ouvrage est encore exploité d'une quelconque manière. Le montant des droits est donc sous-estimé. Des décalages entre les déclarations des éditeurs peuvent aussi être induits par le versement de redevances liées, comme les droits d'accès pour prendre une photographie.

\section{Les droits d'auteur en quelques chiffres}

Le tableau 1 pourrait donner à penser que les droits d'auteur évoluent beaucoup plus vite que le chiffre d'affaires, alors que, en fait, ce phénomène reflète surtout une amélioration des déclarations faites par les éditeurs. Les valeurs indiquées à partir de 2004 se rapprochent donc fortement de la réalité. Si l'on tient compte des maisons d'édition non intégrées dans l'échantillon du SNE, on peut estimer que les droits d'auteur versés par les maisons d'édition françaises s'élèvent à environ un demi-milliard d'euros en 2005. Toutefois, ce chiffre comprend les droits versés à tous les détenteurs des droits d'exploitation, qu'ils soient français ou étrangers, auteurs ou éditeurs, aboutissant ainsi à des doubles comptes, en particulier en raison des cessions de droits en format de poche. Nous estimons ces derniers à 30 millions d'euros maximum.

Tous ces droits ne correspondent pas à un strict travail d'auteur : c'est le cas pour les usages particuliers du droit forfaitaire, les droits des directeurs de collection ou d'ouvrage (pour la fonction apporteur d'ouvrage) ou les droits des packagers. Les montants en jeu sont donc difficiles à estimer, mais, en fonction des usages connus, nous les estimons

6. Aperçu statistique des industries culturelles, Paris, Ministère de la Culture et de la Communication, coll. «Les notes statistiques du DEPS » $\mathrm{n}^{\mathrm{o}}$ 16, janvier 2006. Téléchargeable sur le site du deps http://www.culture.gouv.fr/deps (rubrique «Statistiques »).

7. L'Agessa, association chargée d'une mission de gestion pour le compte de la sécurité sociale et placée sous la double tutelle du ministère des Solidarités, de la Santé et de la Famille et du ministère de la Culture et de la Communication, sert de passerelle entre les auteurs et les caisses primaires d'assurance-maladie pour déterminer les conditions d'affiliation au régime spécifique créé par le législateur en 1975, et faire assurer le service des prestations dues aux affiliés et la délivrance de la carte d'assuré social. 
entre 50 à 100 millions d'euros, le chiffre le plus probable étant de 60 millions.

La concentration des droits sur quelques personnes reste limitée dans l'édition de livres. Une estimation des droits versés sur les dix-huit livres vendus à plus de 100000 exemplaires en 2005 montre que ces best-sellers n'ont pas occasionné plus de $4 \%$ des droits de l'année.

Les cessions de droits, elles, sont sous-estimées par les enquêtes du SNE. D'après nos propres estimations, établies à partir des usages des plus grands acteurs, leur valeur devrait se situer entre 150 et 200 millions d'euros.

Quant aux systèmes de rémunération collective, ils devraient représenter pour les auteurs aux alentours de $10 \%$ de l'ensemble des droits perçus à moyen terme.

\section{INTERACTIONS ENTRE ÉVOLUTIONS DES MODES DE GESTION ET RÉMUNÉRATION DES AUTEURS}

La gestion des maisons d'édition a profondément évolué ces trois dernières décennies en raison de l'usage des outils de gestion et des changements dans les structures capitalistiques du secteur. Ces évolutions, elles-mêmes favorisées par l'informatisation qui permet des analyses projet par projet, influent sur la façon de considérer les droits d'auteur.

\section{Outils de gestion et droits d'auteur sur la vente des livres au détail}

Le calcul de l'investissement ou du risque global - outil de gestion le plus fruste - pousse à privilégier les titres d'auteur, c'est-à-dire ceux qui sont rémunérés en droits proportionnels : cela concerne en particulier les ouvrages de texte, où le travail éditorial est limité. En revanche, le calcul du seuil de rentabilité peut, dans certaines circonstances, exercer une forte pression à la baisse du taux de droits d'auteur, jusqu'à favoriser les œuvres collectives, c'est-à-dire les œuvres qui sont sans droits d'auteur proportionnels.

Plus généralement, les outils de gestion introduisent une vision globale de l'économie d'un livre où chaque poste peut interagir avec un autre. Dans cette optique, le livre ne représente pas qu'un investissement mais un système économique. En intro- duisant les droits d'auteur proportionnels comme une variable et non comme une donnée, le calcul du seuil de rentabilité et l'établissement d'un compte d'exploitation font que le responsable éditorial ne raisonne plus, comme avec la méthode du coefficient, en termes de résultante d'éléments globaux. Les droits d'auteur ne sont donc plus un paramètre externe répercuté sur le prix de vente quitte à augmenter le tirage, mais une variable interne sur laquelle agir.

Au nombre des paramètres avec lesquels le responsable éditorial essaie de jouer, figurent les droits d'auteur, qu'ils soient calculés proportionnellement aux ventes ou forfaitairement, puisqu'une grande part de la constitution du prix du livre échappe à son action. Les coûts sur lesquels il ne peut pas vraiment agir constituent environ les deux tiers du prix si l'on additionne frais et remises liés à la distribution et frais de structure. En effet, les premiers (frais de diffusion-distribution) représentent une part fixe de l'allocation de la valeur du livre pendant toute la durée du contrat, soit au moins trois ans. Quant aux seconds (frais de structure), ils sont fixes et souvent réduits au minimum pour les petites maisons. Enfin, le poids des frais de fabrication, hormis pour les beaux livres, ne varie que très peu et varie en fait surtout en fonction du tirage sur les premiers milliers d'exemplaires. Les droits d'auteur représentant ainsi un des rares postes sur lesquels les éditeurs peuvent agir à court terme, leur tentation de peser sur eux est donc forte.

\section{Outils de gestion et droits d'auteur sur les autres exploitations}

Le responsable éditorial prend en compte également les produits d'autres exploitations que la vente au détail et donc les droits afférents. Dans un univers où la concurrence interne à la filière et externe avec tous les autres produits ou services culturels se développe, l'élasticité des prix n'est en effet pas totale et une maison d'édition ne peut donc plus se contenter d'augmenter ses prix car ceux-ci dépendent plus fortement qu'avant du positionnement commercial. Les outils de gestion tendent ainsi au renforcement du rôle de l'éditeur.

Enfin, l'exemple de la coédition montre que des clauses contractuelles peuvent encourager le développement et la structuration de la production éditoriale dans le cas des livres illustrés. Quant à la coproduction, elle peut jouer le même rôle mais, en renversant le raisonnement précédent, elle peut 
aussi permettre de limiter la rémunération des auteurs pour les autres types de livres.

S'il est difficile de vérifier si les droits tendent à baisser, on peut cependant considérer que les tarifs restent stables en monnaie courante pour les droits forfaitaires ou semi-forfaitaires, comme la rémunération des traductions, et donc régressent en euros constants. Les à-valoir ne croissent pas non plus, sauf pour les best-sellers : des montants de 1500 à $3000 €$ (ou l'équivalent en francs) sont toujours évoqués depuis une vingtaine d'années. Si les taux semblent avoir baissé, c'est plus apparent que réel. En effet, à la fin des années 1990, des jurisprudences ont amené les éditeurs ${ }^{8}$ à limiter les abattements sur les bases de calcul et en particulier la passe $^{9}$. Ce sont des taux réels (au moins plus proches de la réalité, des abattements étonnants subsistant) qui apparaissent maintenant dans les contrats.

\section{Une part du travail éditorial déportée vers l'auteur}

Que leur rémunération soit stable ou en baisse en monnaie constante, les auteurs, en contrepartie des droits, réalisent un travail plus important qu'il y a environ vingt ans grâce aux évolutions techniques. La numérisation du processus a en effet supprimé un certain nombre d'opérations dans le prépresse $^{10}$ : la saisie du texte par l'éditeur ou un prestataire est devenue rare, l'auteur entrant directement son texte avec une feuille de style, voire dans une maquette prédéfinie ou dans un système d'informatique éditoriale incluant SGDB ou XML ; les logiciels de correction, intégrés dans le traitement de texte de l'auteur ou chez l'éditeur, réduisent cette phase. Une partie du travail a donc été déportée vers l'amont, à savoir l'auteur, lequel ne perçoit pour autant aucune rémunération en complément. Ces évolutions ont favorisé depuis le milieu des années 1990 la baisse du prix des livres puisque, en rémunérant les auteurs proportionnellement au prix public tout en leur transférant une partie de leur travail, les éditeurs peuvent réaliser cette baisse plus facilement.

\section{DROITS D'AUTEUR}

\section{ET MODÈLES ÉCONOMIQUES}

\section{SELON LES SECTEURS ÉDITORIAUX}

La description des pratiques contractuelles générales ne permet pas d'appréhender la réalité de la relation entre droits d'auteur et économie du livre, car cette filière est très hétérogène. Les pratiques varient selon les secteurs, qu'il s'agisse du taux de droits, de la rémunération sous forme de droits forfaitaires ou proportionnels, du paiement des à-valoir et de leur montant. En outre, ces variations apparaissent d'un livre à l'autre dans un même secteur éditorial.

Plusieurs constats peuvent être faits.

- Tout d'abord, la pratique de l'à-valoir n'est pas systématique. Le versement d'un à-valoir significatif (plus de $5000 €$ ) concerne un nombre limité de livres. Du point de vue de la gestion, il faut distinguer entre la couverture de l'à-valoir et sa rentabilisation: un titre, en effet, peut être rentable économiquement sans que l'à-valoir soit couvert (voir tableau 2).

- Les droits forfaitaires concernent de nombreux titres, mais sont souvent payés dans le cadre d'œuvres composites. L'œuvre collective, où tous les auteurs sont rémunérés en droits d'auteur for-

\section{Tableau 2 - Couverture et rentabilisation d'un à-valoir en littérature générale}

\begin{tabular}{|lrrr|}
\hline À-valoir & $5000 €$ & $30000 €$ & $100000 €$ \\
Prix public $\mathrm{HT}^{*}$ & $20 €$ & $20 €$ & $20 €$ \\
Remise diffusion-distribution & $53 \%$ & $53 \%$ & $53 \%$ \\
Taux moyen de droits & $10 \%$ & $12 \%$ & $15 \%$ \\
Frais de fabrication unitaire & $1 €$ & $0,9 €$ & $0,8 €$ \\
$\begin{array}{l}\text { Promotion } \\
\text { Frais de structure }\end{array}$ & $4 \%$ & $4 \%$ & $4 \%$ \\
$\begin{array}{l}\text { Seuil de couverture } \\
\quad \text { de l'à-valoir }\end{array}$ & $15 \%$ & $15 \%$ & $15 \%$ \\
$\begin{array}{l}\text { Seuil de rentabilisation } \\
\quad \text { sans frais de structure }\end{array}$ & 6500 ex. & 12500 ex. & 33300 ex. \\
$\begin{array}{l}\text { Seuil de rentabilisation frais } \\
\quad \text { de structure compris }\end{array}$ & 1100 ex. & 6400 ex. & 12800 ex. \\
\end{tabular}

* Ce prix public hors taxe de $20 €$ correspond au prix moyen des vingt meilleures ventes 2005 (Livres hebdo $\mathrm{n}^{\circ}$ 637).

\footnotetext{
8. C'est le cas en particulier de Hachette livre.

9. Rappelons que la passe, un abattement sur quantité à la discrétion de l'éditeur, représentait près de $10 \%$ des quantités vendues à la fin des années 1990.

10. Phase qui va jusqu'à la préparation de la forme imprimante (plaque offset ou rouleau polymère) qui est placée sur les presses à imprimer.
} 
faitaires, est un modèle d'usage relativement limité. Par les montants mis en jeu, elle devrait concerner les éditeurs dont les finances sont les plus solides.

- Pour une part non négligeable des livres (sciences humaines et sociales, sciences dures, scolaire, livres professionnels ou pratiques), la publication entre dans un raisonnement multifactoriel pour les auteurs. Le livre n'est qu'un élément d'une stratégie professionnelle plus globale de valorisation : par exemple, un simple outil promotionnel d'un consultant pour les livres professionnels, ou d'un professionnel vers le grand public pour le livre pratique. Ce peut être aussi la transformation d'un capital symbolique en capital financier dans le scolaire ou la condition essentielle d'une carrière en sciences dures ou en sciences humaines et sociales. Les secteurs concernés correspondent à environ $40 \%$ du chiffre d'affaires de l'édition et du nombre de titres publiés selon le $\mathrm{SNE}^{11}$ (et près de $50 \%$ des titres selon Électre). Certes, tous les auteurs n'ont pas cette démarche, en particulier pour les livres pratiques, mais elle doit être prise en compte dans l'économie de la filière.

Quand le livre n'est qu'une composante de la stratégie professionnelle de l'auteur, l'éditeur détient des arguments majeurs dans la négociation. Le premier de ces arguments est de ne faire aucun lien entre le travail demandé et son indemnisation: l'éditeur ne verse donc pas d'à-valoir ou verse seulement un montant symbolique, lequel n'a pour fonction que de valider l'engagement contractuel. Également en position de force pour négocier les taux de droits, il minimise le taux pour les premières ventes jusqu'à ce que le point mort du livre soit atteint, quitte à lâcher du lest sur la dernière tranche au cas où le livre serait un vrai succès dont tous, éditeur ou auteur mais aussi distributeur, vont bénéficier. Certains éditeurs sont allés au bout de cette logique en ne rémunérant pas les premières ventes.

- Par ailleurs, pour une part non négligeable des titres n'entrant pas dans ces stratégies professionnelles, les auteurs sont dans une recherche de gratification symbolique : leur création est publiée, ils apportent leur contribution au débat d'idées... Une partie des «petits » éditeurs vit sur cette démarche. Ceci entraîne les mêmes conséquences négatives sur la rémunération des auteurs.
- Certains de ces petits éditeurs tiennent des raisonnements proches de ceux de leurs auteurs : ils considèrent leur activité d'édition comme un élément d'une stratégie professionnelle qu'ils valorisent par d'autres prestations : par exemple des interventions en formation ou des animations. Dans ce cas de figure, les livres sont une carte de visite pour pénétrer un marché comme celui des collectivités locales.

\section{Droits D'AUTEUR}

\section{ET GESTION FINANCIÈRE DES MAISONS D'ÉDITION}

Constatant que les paiements sous forme d'àvaloir sont finalement faibles et que les droits dus sur les ventes sont versés fort tard dans le cycle d'exploitation d'un livre, le paramètre « délai de paiement » a été introduit pour compléter l'analyse. Le rôle des droits d'auteur peut ainsi être très contrasté dans les finances des maisons d'édition.

Pour de nombreux éditeurs qui publient des livres en une seule couleur, l'auteur est le principal financier de l'exploitation. C'est lui qui fournit la matière et qui réalise une partie du travail de transformation, mais il ne récupère la contrepartie qu'une fois tous les autres participants au cycle éditorial rémunérés. Ce peut être dans certains cas plusieurs années après la mise en vente du titre, parfois jamais. Dans les pays anglo-saxons, où les arrêtés de droits sont plus fréquents - au moins deux fois par an -, ce phénomène anormal est beaucoup moins sensible.

Pour certains, en revanche, la question du financement des à-valoir se pose avec acuité. Pour y faire face, l'adossement à un groupe solide financièrement devient, si ce n'est une nécessité, du moins un

\section{Tableau 3 - Contribution des droits d'auteur au financement de l'exploitation}

\begin{tabular}{|ll|}
\hline$++\quad$ & Petits éditeurs indépendants d'ouvrages de texte \\
+ & Éditeurs d'ouvrages illustrés « d'auteur » \\
& Éditeurs de littérature générale, \\
& filiales (ou départements) de groupes, \\
& éditeurs de bandes dessinées \\
& Éditeurs d'ouvrages collectifs de référence
\end{tabular}

11. Les livres parascolaires et les guides touristiques, qui sont moins concernés par ces logiques, n'ont pas été inclus. 
atout. D'autres cherchent à retarder les paiements des droits dus sur les ventes par divers moyens, dont la provision pour retours d'invendus est probablement la plus emblématique.

Si les éditeurs d'ouvrages de référence collectifs rencontrent le même problème de financement, le paiement sous forme de droits forfaitaires leur permet une rentabilisation plus forte à terme puisque les auteurs n'ont pas partagé les risques éditoriaux.

Ainsi, pour une majorité d'éditeurs, les droits d'auteur ne sont pas un problème financier, mais une solution. Cependant, une partie des «grands » éditeurs - grands en termes de volume d'affaires est confrontée au phénomène opposé.

Cette question de la rentabilisation de l'investissement en droits sous forme d'à-valoir pousse à porter une attention particulière aux cessions de droits qui constituent, dans ce cas, un des meilleurs moyens d'améliorer de manière significative les résultats.

À cet égard, la profession lutte en permanence pour limiter le rôle des agents. Outre le risque de croissance des taux et des à-valoir, ces nouveaux intervenants limiteraient la rentabilité des exploitations secondes (droits gérés par les auteurs, répartition plus favorable pour ces derniers). Ils pousseraient aussi à réduire les délais de paiement, mettant alors en danger le financement du cycle d'exploitation.

\section{Conclusion}

Cette étude confirme que les droits d'auteur sont un point nodal de l'économie de l'édition de livres mais ne sont pas le moyen unique de rémunération de la création originale puisque celle-ci peut être payée sous forme de salaire ou de prestation de services. Par ailleurs, la rémunération sous forme de droits concerne également des activités connexes à la création, et ce pour une part non négligeable puisqu'elle s'établit entre $10 \%$ et $20 \%$ des droits calculés ${ }^{12}$.

C'est un système qui constitue un cadre économique particulièrement favorable aux maisons d'édition, en particulier grâce à la signature presque systématique d'un contrat de cession portant sur l'ensemble des droits sans contrepartie d'exploitation. L'éditeur a une obligation de moyens mais pas de résultat.

En fait, l'équilibre n'est atteint pour l'auteur qu'en raison de l'existence du droit moral ${ }^{13}$. Seul le droit moral permet à l'auteur d'exercer un vrai droit de regard, mais ses différents attributs permettent également à l'auteur de protéger ses intérêts économiques. Sont dans ce cas, par exemple, le contributeur d'une œuvre collective ou de collaboration lors d'une réédition, ou l'auteur lors d'une cession qu'il peut contester pour mieux faire respecter ses intérêts patrimoniaux. Mais c'est la dialectique droit patrimonial/droit moral qui tient le système car si l'on n'a pas de droit financier, on aura beaucoup de difficultés à faire valoir son droit moral ${ }^{14}$.

L'exemple des coéditions éclaire l'impact des clauses contractuelles sur la production. Les contrats de coédition fondés sur un prix de vente «droits inclus » couplé à un calcul des droits de l'auteur sur ce prix favorisent la pratique de la coédition et contribuent ainsi à l'internationalisation et au développement de la production dans le domaine des livres illustrés.

Pour de nombreuses entreprises d'édition, surtout les plus fragiles, l'auteur joue le rôle de financier de l'activité. Le partage des risques, qui est un des fondements de l'activité éditoriale est alors, dans les faits, très déséquilibré. L'auteur, comme coproducteur du livre, est le dernier à en percevoir les revenus même si, aujourd'hui plus qu'hier, il participe à sa réalisation matérielle. Le souhait/ besoin d'être publié - les raisons pouvant être d'ordre artistique ou narcissique ou professionnel permet ce partage inégal. L'importance de ce dernier facteur ne doit pas être négligée.

Une part des auteurs souhaite se passer de cet intermédiaire grâce aux technologies de la communication: quitte à participer plus en aval dans la chaîne de production, autant tenter de maîtriser toute la chaîne. Cependant, le livre, objet physique, représente une onction nécessaire pour nombre d'auteurs, les éditeurs conservent donc une carte maîtresse, le poids symbolique de l'objet et du label apposé.

12. La filière dont l'économie repose sur le domaine public n'a pas pu être évaluée faute d'informations détaillées sur le chiffre d'affaires des éditeurs, alors que cet aspect est non négligeable pour le secteur du livre au format de poche et participe à sa rentabilité.

13. Xavier GREFFe, Économie de la propriété artistique, Paris, Economica, 2005.

14. Propos exprimés par Christian Roblin à propos des exploitations autres que le support papier, lors d'un entretien le 30 décembre 2004. 
Ces évolutions techniques et organisationnelles poussent aussi à la croissance de la production. Les discussions sur la nature de la production de l'auteur - produit de son travail ou propriété - doivent donc intègrer que sa prestation a évolué puisqu'il effectue un véritable travail de mise en forme matérielle, parfois très avancée, qui mérite une rémunération spécifique.

Les maisons d'édition ont un autre atout, même s'il comporte plus de risques aujourd'hui : ce sont des professionnels du rapport avec le public et de l'exploitation des dérivés de la création. Le livre, dans certains secteurs, n'est qu'une des pièces d'une offre générale de services. Les éditeurs juridiques ou professionnels en sont l'avant-garde quand ils mêlent livres, feuillets mobiles sur abonnement, cédéroms, services web, prestations de conseil et de formation, etc. Se pose alors la question de la rémunération de la création. Dans ce cadre, la tentation d'aller vers l'œuvre collective devient forte. Cependant, au regard du rôle que le livre joue pour certains auteurs et du partage des risques entre ces derniers et les éditeurs, cette tentation devrait rencontrer une forte résistance.

Au titre des risques pesant sur les maisons d'édition, l'histoire récente de la filière montre que l'évo- lution technique, couplée à des changements sociologiques, a laminé un secteur entier de l'édition, celui des encyclopédies en plusieurs volumes. Dans ce contexte, certains éditeurs n'ont trouvé comme solution que de se transformer en prestataires des portails sur internet en leur fournissant de la matière éditoriale.

La rémunération des exploitations qui passent par des instances collectives risque ainsi de devenir cruciale. Celle-ci pourrait représenter $10 \%$ de l'ensemble des droits à moyen terme. Leur potentiel de croissance est cependant lié à la mise en place de mécanismes complémentaires comme, par exemple, une forme ou une autre de licence légale que le législateur a écartée, au moins pour le moment.

Cependant, ce type de conception de l'exploitation d'un livre n'est pas possible pour tous les éditeurs ou pour tous les ouvrages, soit que les premiers n'en aient pas les moyens, soit que les seconds ne s'y prêtent pas. Certains éditeurs ou auteurs adaptent ce type de démarche à leur situation. Ainsi, la publication de livres permet de (ou oblige à) développer d'autres activités comme les animations pour les auteurs d'ouvrages pour la jeunesse ou constitue un outil de promotion pour des éditeurs en région. 


\section{Méthodologie}

La première phase de cette étude a été une phase de description/décryptage de l'organisation de la filière et des pratiques contractuelles générales afin de poser un cadre dans lequel se développent des pratiques spécifiques. Elle a été menée à partir d'une revue bibliographique des ouvrages et thèses récentes sur le sujet.

Ensuite, le travail a porté sur l'évaluation des flux de droits concernés en essayant de recouper les informations existantes, en particulier celles provenant du SNE.

Puis l'accent a été mis sur les droits d'auteur dans l'économie des livres à travers trois types d'étude :

- une analyse de l'interaction potentielle entre mode de gestion des projets éditoriaux et rémunération des auteurs : en quoi tel ou tel type d'outil de gestion peut influer sur les taux de droits, la rémunération au forfait ou non et les à-valoir?

- une description des conséquences des particularités de chaque secteur éditorial, de chaque type de livre sur la rémunération des auteurs afin de préciser l'analyse des pratiques et de voir les modes de rentabilisation, l'impact des droits d'auteur sur l'économie de livres particuliers;

- après cette approche titre par titre, l'étude de ce que représentent les droits d'auteur dans la gestion financière des maisons d'édition a été menée afin de mieux apprécier leur contribution à l'économie générale de la filière.

Ce travail s'est heurté à un écueil de taille, à savoir que la rémunération effective des auteurs est considérée comme un des éléments les plus confidentiels d'une maison d'édition*. Or le nombre de nouveautés et nouvelles éditions paraissant annuellement interdit de vérifier les assertions habituelles. II a donc fallu se contenter d'informations parcellaires recueillies dans des contrats ou lors d'entretiens avec des responsables éditoriaux ou administratifs des différents secteurs de l'édition de manière informelle, ou encore auprès d'auteurs. Par ailleurs, un certain nombre d'acteurs repré- sentant des instances de la profession, en particulier les responsables des sociétés de gestion collective, ont été rencontrés.

Que les informations proviennent des documents ou des personnes, tous les types de structure sont représentés: entreprises à vocation grand public ou s'adressant aux professionnels, maisons d'édition spécialisées dans l'édition de texte ou dans les ouvrages illustrés, petits éditeurs indépendants ou maisons (départements ou filiales) faisant partie de groupe nationaux ou internationaux, entreprises récentes ou anciennes.

L'expérience de formateur et de conseil en gestion des maisons d'édition ainsi que les mémoires du master d'édition de l'université Paris 13 ont également nourri ce travail.

Les informations sur les clauses contractuelles, qui ont alimenté cette étude, concernent un très grand nombre de titres : informations détaillées pour plus d'une centaine d'entre eux et plus générales pour plusieurs dizaines. Les secteurs les plus représentés sont la littérature générale, les livres pratiques, les beaux livres, le scolaire et les sciences humaines et sociales.

Soulignons par ailleurs que le portefeuille contractuel d'un éditeur est le résultat de son histoire, et qu'à ce titre, les livres qui paraissent en 2007 ont, pour certains, été signés il y a plusieurs années. Ce phénomène explique probablement une grande inertie dans les pratiques. Dans cette étude, n'ont été retenus que les titres lancés ou parus depuis l'an 2000.

Dernier point, d'ordre méthodologique, lorsque l'on étudie l'édition de livres : la première précaution à prendre est de sortir du paradigme de la littérature, car les pratiques de ce secteur sont très médiatisées et apparaissent souvent comme les standards.

* L'étude faite par la Commission européenne sur ce sujet à l'occasion de
l'acquisition d'Éditis par Hachette est probablement la seule source qui pour-
rait nous donner une vision précise puisque les réponses étaient obligatoires. 


\section{RÉSUMÉ}

Au-delà du demi-milliard d'euros versés annuellement par les éditeurs français en droits d'auteur, ceux-ci sont un point nodal de l'économie éditoriale. Une pression s'exerce sur leur niveau car c'est le seul paramètre sur lequel un éditeur peut jouer aisément. Les évolutions techniques risquent donc de pousser l'auteur, qui a accru sa part du travail dans la chaîne éditoriale, à prendre en charge l'ensemble de celle-ci, mais le statut du livre reste un atout stratégique des maisons d'édition. La croissance des rémunérations passant par des instances collectives est liée à la mise en place de mécanismes adaptés.

\section{ABSTRACT}

Authors royalties, half billion euros paid by French publishers a year, are a key issue for the book publishing business. They tend to be squeezed because the level of royalties is the only parameter easily manageable by publishers. These are at risk of being outflanked because the authors whose part of the editing process has increased due to technical evolutions, will deal without them. Nevertheless, the book's status remains a key asset for publishers. Besides, the growth of the returns coming through collective institutions is linked to the adaptation of these mechanisms.

Dans la série «Économies des droits d'auteur », sont disponibles :

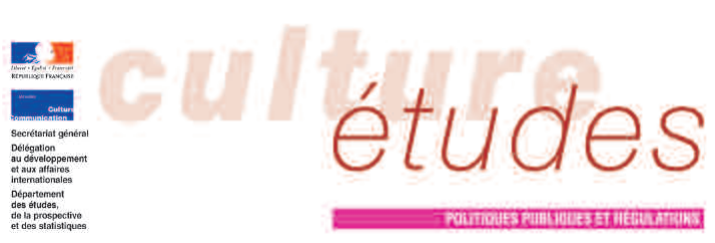

2007-4 I. Le livre

2007-5 II. Le cinéma

2007-6 III. La télévision

2007-7 IV. La photographie

2007-8 V. Synthèse 